\title{
Biodiversity and Seasonal abundance of Mites Associated with Two Varieties of Date Palm in Giza and Sohag Governorates, Egypt
}

\author{
Mariam A. El-Sanady and Azza A. Mohamed \\ Plant Protec. Res. Institute, Agric. Res. Center, Dokki, Giza. Egypt
}

\begin{abstract}
A large number of mites are known associated with different varieties of date palm throughout the world. Their distributional pattern is, however not constant everywhere, which varies according to climatic factors. These mite species could be either Phytophagous, Parasitic, Predators, Phoresy in addition to Saprophagous and Fungivorous species. In this study 37 mite species representing 31 genera, 17 families, three Sub-orders, Actinedida; Gamasida and Acaridida were recorded. These mite species were classified according to their feeding habits into four categories; plant feeders of both leaves and fruits, parasitic and predaceous that play a role as biocontrol agents of different insect and mite pests and fungivorous as well as those of uncertain feeding behavior. The population fluctuation of the phytophagous mites; Eutetranychus orientalis; Oligonychus afrasiaticus (Tetranychidae); Raoiella indica; Phyllotetranychus aegyptiacus Sayed (Tenuipalpidae) on Sewi variety was higher than on Zaghloul variety as well as in Giza than in Sohag during the two seasons, 2010 and 2011. The population of predaceous and parasitic mites associated with different pests infesting date palm increased as well as the population of pests increased. Therefore, the biocontrol agents suppress the populations of different pests in both varieties of date palm in the two localities
\end{abstract}

Key Words: Date palm, Mites, Phytophagous, Predaceous.

\section{INTRODUCTION}

Date palm (Phoenix dactylifera L.) production is a world agricultural industry that produced atout 4.7 million tones of fruit in 1997. The date fruit, which is produced largely in the hot arid regions of southern Asia and North Africa, is marketed all over the world (FAO, 1998). Date palm fruits produced in Egypt are considered the best date fruit varieties, which can be exported to foreign markets provided that the product qualities are most satisfactory, being free from infestation of pests and residues of pesticides, El-Dakroury et al., 2002.

The date palm and its fruits are subjected to attacks by several insect and mite pests that are in most cases well adapted to the Oasis environment. Damage caused by pests is considerable and lead to economic losses.

Biodiversity of mites associated with palm trees, phytophagous, predaceous, parasitic, fungivorous, phoretic and saprophagous are very important to throw lights on.

The present work aims to study the biodiversity and seasonal abundance of the economic mite pests which cause great damage to palm trees and associated predaceous, parasitic mites on Zaghloul and Sewi varieties in Giza and Sohag governorates.

\section{MATERIALS AND METHODS}

An area of two feddans in both Giza and Sohag governorates cultivated with Zaghloul and Sewi varieties of about ten years old; feddan for each variety and place was examined during the period from April to August 2010 and 2011. Samples of 15 leaflets from three trees for each variety were collected, at fortnight intervals exchanged between Giza and Sohag during the period of study from April to August2010 and 2011. Collected samples were transferred to the laboratory for examination using stereomicroscope.

The population fluctuations of different mite species were recorded during period of study for both varieties in the two localities of Giza and Sohag governorates. Speciemens of 2-3 individuals for each mite species were mounted on glass slides using Hoyer,s medium for examination. Mounted mite species were identified according to review given by Hughes, 1976 and Zaher, 1984\& 1986.

\section{· RESULTS AND DISCUSSION}

In this study 37 mite species belonging to 31 genera, representing 17 families of the three suborders, Actinedida, Gamasida and Acaridida were collected. These mite species were classified according to their feeding behavior to four groups as follows:

\section{I- Phytophagous mites:}

\section{A- Suborder: Actinedida}

\section{1- Family: Tetranychidae Donnadeiu}

The date palm leaf brown mite Eutetranychus palmatus Attiah (1967) causes injury to leaf date palm trees. This mite species is feeding on upper leaf surface produces a multitude of gray spots, 
which giving leaves a chlorotic appearance. The infested leaves become weaken and finally drop. This mite species was recorded in rare numbers in Giza and Sohag governorates in 2010 then increased during 2011 to moderate level.

The date palm mite, Oligonychus afrasiaticus (McGregor) was collected in moderate numbers on both Zaghloul and Sewi varieties during 2010 in Giza; while its population was rare on Zaghloul and moderate on Sewi. On the other hand, during 2011, Sewi variety infested with in high numbers in Giza, table 1. Field observation showed heavy deposit of fine webs collecting dust. This mite species feeds long the midrib on lower surface of leaves causing yellowish patches at the points of attack. The mite also feeds on dates producing scar tissues on date skin, causing harden cracks and shrivel with subsequent reduction in fruit grade marketing. Population of mites increased during July and August, tables $5 \& 6$.

\section{2- Family: Tenuipalidae Berlese}

The red palm mite, Raoiella indica Hrist which also, known as the coconut mite lives on the underside of the leaves of the host plant in very large numbers. All active stages of the mite are dark red with black markings and attacked leaves display severe yellowing. Zaher et al., 1984 studied the biology of Phyllotetranychus aegyptiacus Sayed infesting date palm trees the U.A.R.

The population of $R$. indica during this study showed that the botl: Zaghloul and Sewi varieties had generally moderate levels of infestation in Giza during 2010; while in 2011 Zaghloul variety in Sohag had in tables 1, 5 \& 6. Pena et al., 2006 reported the red palm mite $R$. indica to be an important pest of coconut and date palms. Flechtmann and Jean Etienne 2004 reported that $R$. indica threatened palms in the Americas. Pena et al., 2006 reported the red palm mite $R$. indica to be an important pest of coconut and date palms. Flechtmann and Jean Etienne 2004 reported that $R$. indica threatened palms in the Americas.

Phyllotetranychus aegyptiacus Sayed was recorded in high numbers on leaves of both varieties during the season 2010 and 2011 in the two localities except on Zaghoul variety in Giza in 2010 that had moderate level of infestation.

The infestation of $P h$. aegyptiacus symptoms appears different from that of $R$. indica by white blotches due to the aggregation of mites with white fanlike setae. The heavy mite infestations produce sufficient webbing. Our study proved its occurrence in high numbers on the two varieties in the two localities and years except on Zaghloul in Giza in 2010 (tables 1, 5 \& 6).

\section{3- Family: Tarsonemidae Kramer}

Some sprcies of tarsomemid mites become serious pests on different crops. The two mite species, Polyphagotarsonemus latus (Banks) and Stenotarsonemus spirifix March were recorded on Zaghloul and Sewi varieties in Giza and Sohag governorates, table 1. El-Dakroury et al., 2002 montioned that date palm was infested with so many insect and mite pests.

\section{II - Predaceous mites: \\ Suborder: Gamasida \\ 1- Family: Phytoseiidae Berlese}

The Phytoseiid mites were represented by two predators associated with different pests infesting date palm trees.

- Euseius scutalis A.-H. was recorded on both date palm varieties in few numbers in Giza and Sohag localities

- Amblyseius swirskii (A.-H.) was found in moderate numbers in Giza and high numbers in Sohag on both varieties

\section{2- Family: Ascidae Voigts \& Oudemans}

Four predatory mite species of the family Ascidae were recorded associated with pests infesting date palm trees in both Giza and Sohag localities in rare numbers during the period of study, 2010 and 2011 years, table 2.

\section{3- Family: Laelapidae Berlese \\ - Androlaelaps casalis Berlese \\ - Hypoospis miles Berlese \\ - H. sardoa Berlese}

Three mite speieces were recorded between rarely and moderate numbers on Zaghloul and Sewi variaties in the two localities.

\section{4- Family: Sejidae Berlese}

The predatory mite, Sejius paloghi, the only species of Sejid mites was recorded during 2011 on the two varieties of date palm trees in rare numbers in both governorates.

\section{5- Family: Macrochelidae Vitzthum \\ - Macrocheles carintus Koch \\ - M. ascaedomestica Scopli \\ - Glyptholaspis confusa rao}

The macrochelid mites may play an important role as a bio-control agent suppressing

the different pests populations on different crops, as well as date palm trees. 
Table (1): Incidence of Phytophagous mites infesting date plam trees in Giza and Sohag governorates, Egypt, during 2010 \& 2011

\begin{tabular}{|c|c|c|c|c|c|c|c|c|c|}
\hline \multirow{4}{*}{ Suborders \& Families } & \multirow{4}{*}{ Species } & \multicolumn{8}{|c|}{ Abundance } \\
\hline & & \multicolumn{4}{|c|}{ Giza } & \multicolumn{4}{|c|}{ Sohag } \\
\hline & & \multicolumn{2}{|c|}{2010} & \multicolumn{2}{|c|}{2011} & \multicolumn{2}{|c|}{2010} & \multicolumn{2}{|c|}{2011} \\
\hline & & $Z$ & $\mathrm{~S}$ & $\mathrm{Z}$ & $\mathrm{S}$ & $\mathrm{Z}$ & $\mathrm{S}$ & $\mathrm{Z}$ & $\mathrm{S}$ \\
\hline \multirow{2}{*}{$\begin{array}{l}\text { Actinedida Tetranychidae } \\
\text { Donnadeiu }\end{array}$} & Eutetranychus palmatus Attiah & + & ++ & ++ & ++ & + & ++ & ++ & ++ \\
\hline & Oilgonychus afrasiaticus (MCGregor) & ++ & ++ & ++ & +++ & ++ & + & ++ & ++ \\
\hline \multirow{2}{*}{ Tenuipalpidae Berlese } & Raoiella indica Hirst & ++ & ++ & ++ & ++ & ++ & ++ & ++1 & +++ \\
\hline & Phyllotetranychus aegyptiacus Sayed & ++ & +++ & +++ & +++ & +++ & +++ & +++ & +++ \\
\hline \multirow{2}{*}{ Tarsonemidae Kramer } & Polyphagotarsonemus latus (Banks) & + & ++ & ++ & +++ & +++ & +++ & ++ & +++ \\
\hline & Stenotarsonemus spirifix March & + & ++ & ++ & +++ & ++ & +++ & ++ & +++ \\
\hline $\begin{array}{l}Z=\text { Zaghloul variety } S= \\
++=\text { Moderate }(3-4 \text { indivic }\end{array}$ & $\begin{array}{lr}\text { vi variety } & +=\text { Rare }(1-2 \\
\text { s / inch) } & +\mathrm{H}=\mathrm{Hi}\end{array}$ & & & & & & & & \\
\hline
\end{tabular}

Table (2): Incidence of predaceous mites inhabiting from date palm trees in Giza and Sohag governorates,

Egypt, during 2010 \& 2011

\begin{tabular}{|c|c|c|c|c|c|}
\hline \multirow{3}{*}{ Suborders \&Families } & \multirow{3}{*}{ Species } & \multicolumn{4}{|c|}{ Abundance } \\
\hline & & \multicolumn{2}{|c|}{ Giza } & \multicolumn{2}{|c|}{ Sohag } \\
\hline & & 2010 & 2011 & 2010 & 2011 \\
\hline \multirow{2}{*}{ Gamasida Phytoseiidae Berlese } & Euseuis scutalis A.-H. & + & + & + & + \\
\hline & Amblyseius swirskii (A.-H.) & ++ & ++ & +++ & +++ \\
\hline \multirow{4}{*}{ Ascidae Voigts \&Oud. } & Blattisocius keegani (Fox) & + & + & ++ & ++ \\
\hline & Lasioseius bispinosus Evans & + & + & ++ & ++ \\
\hline & Proctolaelaps pygmaeus Muller & + & + & + & ++ \\
\hline & Melichares ornate Berlese & + & + & + & + \\
\hline \multirow{3}{*}{ Laelapidae Berlese } & Androlaelaps casalis Berlese & + & ++ & + & ++ \\
\hline & Hypoaspis miles Berlese & + & ++ & + & ++ \\
\hline & Hypoaspis sardoa Berlese & + & + & + & \\
\hline Sejidae Berlese & Sejius baloghi (Athias- Henriot) & - & + & - & + \\
\hline \multirow{3}{*}{ Macochoelidae Vitzhum } & Macrocheles carintus Koch & ++ & ++ & ++ & ++ \\
\hline & Macrocheles mascaedomesticae (Scopoli) & + & ++ & ++ & ++++ \\
\hline & Glyptholaspis confusa Fao & + & + & + & + \\
\hline \multirow{2}{*}{ Uropodidae Berlese } & Uropoda minima Kramar & ++ & +++ & ++ & +++ \\
\hline & Chiropturopoda bakeri Zaher\&Afifi & + & + & + & + \\
\hline \multirow{3}{*}{ Actinedida Cheyletidae Leach } & Cheyletus malaccensis Oud. & ++ & ++ & ++ & ++ \\
\hline & Cheyletus fortis Oud. & + & + & ++ & ++ \\
\hline & Cheletogenes ornatus (Can\&Fons.) & + & + & + & ++ \\
\hline \multirow{2}{*}{ Cunaxidae Thor. } & Cunaxa capreolus Berlese & + & + & + & + \\
\hline & Pulaeus zaheri (El-Bishlawi\& Rakha) & + & + & + & + \\
\hline \multirow{2}{*}{ Stigmaeidae Oud. } & Agistemus exsertus Gonz. & ++ & ++ & ++ & ++ \\
\hline & Agistemus africanus Soliman \&Gomaa & + & + & + & + \\
\hline \multirow[t]{2}{*}{ Tydeidae Kramer } & Pronematus ubiquitus McGregor & +++ & +++ & +++ & +++ \\
\hline & Tydeus californicus (Banks) & ++ & +++ & ++ & +++ \\
\hline Acaridida Hemisarcoptidae Oud. & Hemisarcoptes malus (Shimer) & ++ & ++ & ++ & +++ \\
\hline
\end{tabular}

Table (3): Incidence of parasitic mites associated with mite pests infesting date palm trees in Giza and Sohag governorates, Egypt, during 2010 \& 2011

Suborders \& Families

Actinedida Pyemotidae Oud.

Gamasida Uropodidae Berlese

$+=$ Rare (1-2 individuals / leaf)
Species

Pyemotes herfsi (Oud.) Pyemotes tritici (La-Greze-Fossot \& Mantane) Leiodinychus karmoeri (G.\&R.,Canestrini) $++=$ Moderate (3-4 individuals / leaf) $+++=$ High (more than individuals / leaf) 
Table (4): Incidence of fungivorus mites collected from leaflets of date palm trees in Giza and Sohag governorates, Egypt, during 2010 \& 2011

\begin{tabular}{|c|c|c|c|c|c|}
\hline \multirow{3}{*}{ Suborders \& Families } & \multirow{3}{*}{ Species } & \multicolumn{4}{|c|}{ Abundace } \\
\hline & & \multicolumn{2}{|c|}{ Giza } & \multicolumn{2}{|c|}{ Sohag } \\
\hline & & 2010 & 2011 & 2010 & 2011 \\
\hline \multirow{3}{*}{$\begin{array}{c}\text { - Acaridida Acaridae } \\
\text { Leach }\end{array}$} & Tyrophagus putrescentiae (Schrank) & +++ & ++ & ++ & ++ \\
\hline & Tyrophagus entomophagus (Laboulbèn and Robin) & + & ++ & + & ++ \\
\hline & Mycetoglyphus fungivorus Oud. & + & ++ & + & ++ \\
\hline
\end{tabular}

Table (5): Seasonal abundance of phytophagous mites infesting two varieties of date palm in the two governorates Giza \& Sohag in 2010

\begin{tabular}{|c|c|c|c|c|c|c|c|c|c|}
\hline \multirow{3}{*}{\multicolumn{2}{|c|}{ Inspection date }} & \multicolumn{8}{|c|}{ Zaghloul variety } \\
\hline & & \multicolumn{4}{|c|}{ Giza } & \multicolumn{4}{|c|}{ Sohag } \\
\hline & & $\begin{array}{c}E . \\
\text { palmatus }\end{array}$ & $\begin{array}{c}O . \\
\text { afrasiaticus }\end{array}$ & $\begin{array}{c}R . \\
\text { indica }\end{array}$ & $\begin{array}{c}P . \\
\text { egypticus }\end{array}$ & $\begin{array}{c}E \\
\text { palmatus }\end{array}$ & $\begin{array}{c}O . \\
\text { afrasiaticus }\end{array}$ & $\begin{array}{c}R . \\
\text { indica }\end{array}$ & $\begin{array}{c}P . \\
\text { egypticus }\end{array}$ \\
\hline \multirow[t]{2}{*}{ April } & 1 & 12 & 22 & 18 & 26 & 8 & 15 & 16 & 19 \\
\hline & $\overline{15}$ & 16 & 26 & 22 & 28 & 10 & 18 & 21 & 21 \\
\hline \multirow[t]{2}{*}{ May } & 1 & 19 & 39 & 32 & 43 & 13 & 21 & 30 & 28 \\
\hline & 15 & 25 & 42 & 35 & 56 & 15 & 32 & 33 & 33 \\
\hline \multirow[t]{2}{*}{ June } & 1 & 31 & 54 & 44 & 72 & 22 & 35 & 35 & 39 \\
\hline & 15 & 37 & 62 & 51 & 78 & 25 & 41 & 45 & 45 \\
\hline \multirow[t]{2}{*}{ July } & 1 & 42 & 71 & 54 & 85 & 28 & 44 & 49 & 48 \\
\hline & $\overline{15}$ & 53 & 76 & 60 & 94 & 33 & 52 & 55 & 58 \\
\hline \multirow[t]{2}{*}{ August } & 1 & 58 & 85 & 62 & 111 & 38 & 56 & 59 & 57 \\
\hline & 15 & 62 & 104 & 68 & 129 & 48 & 68 & 62 & 55 \\
\hline Total & & 355 & 581 & 446 & 717 & 240 & 382 & 405 & 403 \\
\hline Mean & & $35.5 \mathrm{~b}$ & $58.1 \mathrm{ab}$ & $44.6 \mathrm{~b}$ & $71.7 \mathrm{a}$ & $24 \mathrm{~b}$ & $38.2 \mathrm{a}$ & $40.5 \mathrm{a}$ & $40.3 \mathrm{a}$ \\
\hline \multicolumn{2}{|l|}{ LSD } & \multicolumn{4}{|c|}{22.67} & \multicolumn{4}{|c|}{13.91} \\
\hline \multirow{3}{*}{\multicolumn{2}{|c|}{ Inspection date }} & \multicolumn{8}{|c|}{ Sewi variety } \\
\hline & & \multicolumn{4}{|c|}{ Giza } & \multicolumn{4}{|c|}{ Giza } \\
\hline & & $\begin{array}{c}E . \\
\text { palmatus }\end{array}$ & $\begin{array}{c}O . \\
\text { afrasiaticus }\end{array}$ & $\begin{array}{c}R . \\
\text { indica }\end{array}$ & $\begin{array}{c}P . \\
\text { egypticus }\end{array}$ & $\begin{array}{c}E . \\
\text { palmatus }\end{array}$ & $\begin{array}{c}0 . \\
\text { afrasiaticus }\end{array}$ & $\begin{array}{c}R . \\
\text { indica }\end{array}$ & $\begin{array}{c}P . \\
\text { egypticus }\end{array}$ \\
\hline \multirow[t]{2}{*}{ April } & 1 & 24 & 39 & 35 & 48 & 21 & 18 & 22 & 35 \\
\hline & 15 & 33 & 45 & 38 & 56 & 25 & 21 & 31 & 42 \\
\hline \multirow[t]{2}{*}{ May } & 1 & 38 & 48 & 42 & 75 & 32 & 22 & 35 & 48 \\
\hline & 15 & 41 & 54 & 45 & 68 & 35 & 35 & 39 & 61 \\
\hline \multirow[t]{2}{*}{ June } & 1 & $45^{\circ}$ & 62 & 49 & 85 & 41 & 36 & 45 & 77 \\
\hline & $\overline{15}$ & 35 & 75 & 58 & 94 & 40 & 48 & 44 & 68 \\
\hline \multirow[t]{2}{*}{ July } & 1 & 52 & 78 & 76 & 101 & 42 & 49 & 56 & 74 \\
\hline & $\overline{15}$ & 66 & 87 & 89 & 105 & 48 & 56 & 60 & 84 \\
\hline \multirow[t]{2}{*}{ August } & 1 & 75 & 89 & 98 & 111 & 50 & 68 & 68 & 91 \\
\hline & $\overline{15}$ & 89 & 94 & 115 & 125 & 66 & 75 & 75 & 96 \\
\hline \multicolumn{2}{|l|}{ Total } & 498 & 671 & 654 & 868 & 400 & 428 & 478 & 676 \\
\hline \multicolumn{2}{|l|}{ Mean } & 49.8 & 67.1 & $65.4 \mathrm{~b}$ & $86.8 \mathrm{a}$ & $40 \mathrm{~b}$ & $42.8 \mathrm{~b}$ & $47.5 b$ & $67.6 \mathrm{a}$ \\
\hline \multicolumn{2}{|l|}{ LSD } & & 21.4 & & & \multicolumn{4}{|c|}{16.24} \\
\hline
\end{tabular}

The same letters at the same governorate are not significantly different.

\section{6- Family: Uropodidae Berlese}

Two Uropodid mite species, Uropoda minima Kramer, was recorded in moderate numbers; while Chiropturopoda bakeri Zaher \&Afifi was recorded in rare numbers on both varieties and localities.

\section{C- Suborder: Actinedida}

\section{1- Family: Cheyletidae Leach}

The Cheyletid mites were represented by three specie on Zaghloul and Sewi varieties in both localities in Giza and Sohag during the two years, table 2.

\section{2- Family: Cunaxidae Thor}

- Cunaxa capreolus Berlese

- Pulaeus zaheri El-Bishlawi \& Rakha. These two cunaxid mites were recorded in rare numbers, table 2. 
Table (6): Seasonal abundance of phytophagous mites infesting two varieties of date palm in two governorates Giza \& Sohag in 2011

\begin{tabular}{|c|c|c|c|c|c|c|c|c|c|}
\hline \multirow{3}{*}{\multicolumn{2}{|c|}{$\begin{array}{l}\text { Inspection } \\
\text { date }\end{array}$}} & \multicolumn{8}{|c|}{ Zaghloul variety } \\
\hline & & \multicolumn{4}{|c|}{ Giza } & \multicolumn{4}{|c|}{ Sohag } \\
\hline & & $\begin{array}{c}E . \\
\text { palmatus }\end{array}$ & $\begin{array}{c}O . \\
\text { afrasiaticus }\end{array}$ & $\begin{array}{c}R . \\
\text { indica }\end{array}$ & $\begin{array}{c}P . \\
\text { aegypticus }\end{array}$ & $\begin{array}{c}E . \\
\text { palmatus }\end{array}$ & $\begin{array}{c}O . \\
\text { afrasiaticus }\end{array}$ & $\begin{array}{c}R . \\
\text { indica }\end{array}$ & $\begin{array}{c}P . \\
\text { aegypticus }\end{array}$ \\
\hline \multirow[t]{2}{*}{ April . } & 1 & 19 & 32 & 23 & 55 & 5 & 20 & 32 & 72 \\
\hline & 15 & 29 & 45 & 35 & 59 & 11 & 18 & 39 & 69 \\
\hline \multirow[t]{2}{*}{ May } & 1 & 31 & 48 & 38 & 67 & 13 & 28 & 44 & 85 \\
\hline & 15 & 38 & 53 & 42 & 68 & 18 & 31 & 49 & 88 \\
\hline \multirow[t]{2}{*}{ June } & 1 & 42 & 64 & 55 & 81 & 25 & 38 & 58 & 95 \\
\hline & 15 & 45 & 69 & 64 & 84 & 38 & 49 & 84 & 111 \\
\hline \multirow[t]{2}{*}{$\begin{array}{l}\text { July } \\
\end{array}$} & 1 & 54 & 72 & 63 & 114 & 44 & 50 & 94 & 121 \\
\hline & 15 & 61 & 72 & 68 & 95 & 59 & 63 & 105 & 131 \\
\hline \multirow[t]{2}{*}{ August } & 1 & 68 & 78 & 75 & 108 & 62 & 75 & 111 & 142 \\
\hline & 15 & 75 & 88 & 88 & 145 & 65 & 98 & 122 & 155 \\
\hline Total & & 462 & 627 & 551 & 876 & 340 & 470 & 738 & 1069 \\
\hline Mean & & $46.2 \mathrm{~b}$ & $62.7 \mathrm{~b}$ & $55.1 \mathrm{~b}$ & $87.6 \mathrm{a}$ & $34 \mathrm{c}$ & $47 c$ & $73.8 \mathrm{~b}$ & $106.9 \mathrm{a}$ \\
\hline \multicolumn{2}{|l|}{ LSD } & \multicolumn{4}{|c|}{19.41} & \multicolumn{4}{|c|}{25.49} \\
\hline \multirow{3}{*}{\multicolumn{2}{|c|}{ Inspection date }} & \multicolumn{8}{|c|}{ Sewi variety } \\
\hline & & \multicolumn{4}{|c|}{ Giza } & \multicolumn{4}{|c|}{ Sohag } \\
\hline & & $\begin{array}{c}E . \\
\text { palmatus }\end{array}$ & $\begin{array}{c}O . \\
\text { afrasiaticus }\end{array}$ & $\begin{array}{c}R . \\
\text { indica }\end{array}$ & $\begin{array}{c}P . \\
\text { aegypticus }\end{array}$ & $\begin{array}{c}E . \\
\text { palmatus }\end{array}$ & $\begin{array}{c}O . \\
\text { afrasiaticus }\end{array}$ & $\begin{array}{c}R . \\
\text { indica }\end{array}$ & $\begin{array}{c}P . \\
\text { aegypticus }\end{array}$ \\
\hline \multirow[t]{2}{*}{ April } & 1 & 15 & 28 & 28 & 62 & 18 & 25 & 45 & 168 \\
\hline & 15 & 22 & 44 & 30 & 66 & 21 & 32 & 52 & 188 \\
\hline \multirow[t]{2}{*}{ May } & 1 & 19 & 59 & 40 & 75 & 33 & 38 & 66 & 201 \\
\hline & 15 & 33 & 62 & 45 & 89 & 45 & 41 & 78 & 218 \\
\hline \multirow[t]{2}{*}{ June } & 1 & 41 & 77 & 61 & 32 & 48 & 45 & 89 & 249 \\
\hline & 15 & 48 & 89 & 68 & 99 & 49 & 49 & 94 & 277 \\
\hline \multirow[t]{2}{*}{ July } & 1 & 45 & 94 & 69 & 112 & 50 & 48 & 97 & 312 \\
\hline & 15 & 49 & 98 & 75 & 10 & 52 & 58 & 104 & 345 \\
\hline \multirow[t]{2}{*}{ August } & 1 & 54 & 101 & 88 & 122 & 68 & 65 & 112 & 380 \\
\hline & 15 & 58 & 111 & 96 & 125 & 75 & 78 & 122 & 415 \\
\hline \multicolumn{2}{|l|}{ Total } & 339 & 763 & 600 & 947 & 459 & 479 & 859 & 1140 \\
\hline \multicolumn{2}{|l|}{ Mean } & $38.4 \mathrm{~b}$ & $76.3 \mathrm{a}$ & $60 \mathrm{ab}$ & $94.7 \mathrm{a}$ & $45.9 \mathrm{~b}$ & $47.9 \mathrm{~b}$ & $85.9 \mathrm{~b}$ & $275.3 \mathrm{a}$ \\
\hline LSD & & \multicolumn{4}{|c|}{42.72} & \multicolumn{4}{|c|}{41.76} \\
\hline
\end{tabular}

The same letters at the same governorate are not significantly different.

\section{3- Family Stigmaidae Oudmans}

- Agistemus exsertu: Gonzalis. This predatory mite was found in moderate numbers associated with pests infesting date palm varieties in Giza and Sohag governorates.

- Agistemus afrasiaticus Soliman \& Gomaa. This predatory mite species was recorded in rare numbers on Zaghloul and Sewi varieties in both Giza and Sohag governorates, table 2.

\section{4- Family Tydeidae Kramer}

Two predatory mite speies were recorded, of which, Pronematus obiquitus Mc.G. was found in high numbers; while Tydeus californicus Banks in moderate numbers associated with different pests infesting date palm trees under investigation in both Giza and Sohag governorates, table 2.

\section{D- Suborder: Acaridida.}

\section{1- Family: Hemisarcoptidae, Oudemans}

The predatory mite, Hemisarcoptes malus (Shimer) was recorded in moderate numbers associated with scale insects infesting date palm trees in the two governorates.

El-Halawany et al., 1986 recorded $H$. malus as a predatory mite on scale insets. Sallam et at., 2007 studied the predatory insects, mites and spiders associated with pests infesting date palm in Rashid region, El-Beheira governorate.

\section{III- Parasitic mites:}

The parasitic mites play an important role in controlling some insect and mite pests associated with date palm trees. Al-Dhafar and Al-Qahtani 
2012 recorded three mite species on date palm, one of which was Aegyptus alhassa n. sp. a parasite collected from egg, larva and pupae of the red palm weevil $R$. farrugineus.

In this study the uropodid mite, Leiodinychus karmeori (G. \& R.,Canestrini) was recorded associated with pupae and adults of the red palm weevil $R$. ferrugineus and the two pyemotid mite species, Pyemotes herfici Oud. and P. tritic La G., F. and $M$. were associated with some insects. Hassan et al., 2011 were recorded thirteen mite species associated with adults and pupae of $R$. ferrugineus in Ismailia governorate.

\section{IV- Fungivorous mites:}

Three mite specie: of the family Acaridae were recorded on leaves of the two date palm varieties, Zaghloul and Sewi in both localities Giza and Sohag. These mite species were Tyrophegous putrescentiae found in moderate numbers on two the varieties in the two localities, $T$. entomophagus and Mycetoglyphus fungivorus were rarely found during 2010 and in moderate numbers in 2011.

\section{Population dynamic of phytophagous mites infesting date palm varieties in Giza and Sohag governorates: \\ Abundance of date palm leaf brown mites, Eutetranychus orientalis:}

The abundance (number of mites/inch) of $E$. palmatus Attia on Zaghloul and Sewi varieties was examined during the period from April to Augest in 2010 and 2011. The :nite started in few numbers in April, then increased to the highest in Augest on both Zaghloul and Sewi varieties. As shown in table (5). Sewi variety was infested with the higher population (355.0 \& 240.0 mites) during 2010 .

Considering the population fluctuation of $O$. afriasiaticus Mc.Gregor on the two date palm varieties, Sewi variety had the higher infestation in 2010 \& 2011 in Giza than in Sohag governorates tables $(5 \& 6)$.

The population trend of $R$. indica and Phyleotetranychus aegypticus on the two tested varieties tables $(5 \& 6)$ showed almost similar trend during the two seasons. The population started low then increased gradually till mid of Augest. Obtained results cleared that populations of the phylophagous mites, $\cdot E$. orientalis, $O$. afriaticus, $R$. indica and $P h$. aegyptious were high on Sewi and in
Giza governorate. Zaher et al., 1984 carried out biological studied on the red palm $R$. indica and Phyllotetranychus aegyptiacus infesting date palm trees.

\section{REFERENCES}

Al- Dhafar, Z. M. and Al-Qahtani, A. M. 2012. Mites associated with the red palm weevil, Rhynchophorus ferrugineus (Olivier) in Saudi Arabia with a description of a new species. Acarines, 6: 3-6.

Attiah, H. H. 1967. The genus Eutetranychus in - U.A.R. with description of three new species (Acarina - Tetranychidae). Bull. Soc. Ent. Egypt, 51:7-10.

El-Dakroury, M. S. I.; Eweis, M. A. and Abul Fadl, H. A. A. 2002. Mass production and NH lization of Trichoghanma \& van e scens westw. As a biocontrol agent against date fruit pests in Siwa Oasis, Egypt. $2^{\text {nd }}$ Int. conf. plant prot. Res. Institute., Cairo, Egypt 21-24 December: 363365.

El-Halawany, M. E.; Kandeel, M. H. and Rakha, M. A. 1986. Mites inhabiting deciduous fruit trees. Agric. Res. Rev., 64(1): 115 - 122.

FAO. 1998. FAO production. Year book food and Agric. Örganiz. of united Nation. Rome. 250 pp.

Flechtmann, C. H. W and Jean Etienne. 2004. The red palms mite Raoiella indica Hiset, a threat to palms in the Americas. J. systematic and Applied Acarology, 9: 109-110.

Hassan, M. F.; Nasr, A. K.; Allam, Sally, F. ; H. A. Taha and Reham, Mahmoud, A. 2011. Biodiversity and seasonal punctuation of mite families associated with the red palm weevi (Rhynchophorous ferrugineus Olivier) (Coleoptera: Curculionidae) in Egypt, Egyptian, J. of Bio. Pest control, 21 (2): 317-323.

Hughes, A. M. 1976. The mites of stored food and houses. Ministry of Agriculture, Fisheries and Food, Tech. Bull. No.9. 400 pp.

Sallam, Gihan, M.; Yassin, E. M. A. and Nahla, Abd El-Azeem, I. 2007. The predatory insects, mites and spiders associated with date palm pests in Rashid region, El- Beheria governorate, Egypt. Egypt, J. Agric. Res., 85 (1): 37-51.

Zaher, M. A. 1984. Survey and Ecological studies on phytophagous, predaceous and soil mites in Egypt. Phytophagous mites in Egypt PL. 480 programe U.S.A. project No. EG. ARS, 30. Grant No. FG-EG- 139. 